\title{
Brewer's spent grain from different types of malt: Evaluation of the antioxidant activity and identification of the major phenolic compounds
}

\author{
Manuela M. Moreira, Simone Morais, Daniel O. Carvalho, Aquiles A. Barros, Cristina \\ Delerue-Matos, Luís. F. Guido
}

\begin{abstract}
A B S T R A C T
The antioxidant activity and phenolic composition of brewer's spent grain (BSG) extracts obtained by microwave-assisted extraction from two malt types (light and dark malts) were investigated. The total phenolic content (TPC) and antioxidant activity among the light BSG extracts (pilsen, melano, melano 80 and carared) were significantly different $(p<0.05)$ compared to dark extracts (chocolate and black types), with the pilsen BSG showing higher TPC $\left(20 \pm 1 \mathrm{mg}_{\mathrm{GAE}} / \mathrm{g}\right.$ dry BSG). In addition, the antioxidant activity assessed by 2,2-diphenyl1-picrylhydrazyl, 2,2'-azino-bis(3-ethylbenzothiazoline-6-sulfonic acid) and deoxyribose assays decreased as a result of increasing kilning temperatures in the following order: pilsen $>$ melano $>$ melano $80>$ carared $>$ chocolate $>$ black. HPLC-DAD/ESI-MS/MS analysis indicated the presence of phenolic acids, such as ferulic, $p$-coumaric and syringic acids, as well as several isomeric ferulate dehydrodimers and one dehydrotrimer. Chocolate and black extracts, obtained from malts submitted to the highest kilning temperatures, showed the lowest levels of ferulic and p-coumaric acids. These results suggested that BSG extracts from pilsen malt might be used as an inexpensive and good natural source of antioxidants with potential interest for the food, pharmaceutical and/or cosmetic industries after purification.
\end{abstract}

\author{
Keywords: \\ Brewer's spent grain \\ Microwave-assisted extraction \\ Polyphenols \\ Antioxidant activity \\ Kilning temperature \\ HPLC-DAD/ESI-MS/MS analysis
}

\section{Introduction}

Brewer's spent grain (BSG), the barley malt residue obtained after wort manufacture, is the main by-product from breweries, representing approximately $20 \mathrm{~kg}$ per $100 \mathrm{~L}$ of beer produced (Aliyu \& Bala, 2011). BSG is available at low or no cost all through the year, but its main application has been limited to animal feed. Nevertheless, it is a lignocellulosic material that can be better used, since it is rich in oligo- and polysaccharides as well as in polyphenols (Mussatto, 2009). Several attempts have been made to reuse this material for industrial applications such as the production of alpha-amylase (Hashemi, Razavi, Shojaosadati, \& Mousavi, 2011), activated carbon (Mussatto et al.,
2010), ethanol (White, Yohannan, \& Walker, 2008), lactic acid (Mussatto, Fernandes, Dragone, Mancilha, \& Roberto, 2007) and xylitol (Mussatto \& Roberto, 2005).

In the last few years, numerous research studies have associated the consumption of foods rich in bioactive compounds with the ability to promote a number of benefits for human health. The most common bioactive compounds include secondary metabolites such as antibiotics, mycotoxins, alkaloids, food grade pigments, plant growth factors, and phenolic compounds (Martins et al., 2011; Meneses, Martins, Teixeira, \& Mussatto, 2013; Routray \& Orsat, 2012). Particularly, phenolic compounds are of considerable interest to scientists, manufacturers and consumers due to their importance in food quality, with protective and preventive roles in certain types of cancer and several other chronic diseases (Barbosa-Pereira, Angulo, Paseiro-Losada, \& Cruz, 2013; Krishnaswamy, Orsat, Gariépy, \& Thangavel, 2013; Naczk \& Shahidi, 2004). Hydroxycinnamic acids, which are the predominant phenols in BSG (Bartolomé \& Gómez-Cordovés, 1999; Bartolomé, Santos, Jiménez, del Nozal, \& Gómez-Cordovés, 2002; Mussatto, Dragone, \& Roberto, 2007), have shown antioxidant properties and the in vitro antioxidant effect of these compounds was reported to be similar to that exhibited by the well-known antioxidants $\alpha$-tocopherol and ascorbic acid (McCarthy et al., 2012). Moreover, the low-cost and large availability of BSG, associated with the current interest in the health benefits of 
phenolic acids, opens up new possibilities for the use of this brewery by-product.

Although there is a large amount of information available relating to the health effects of polyphenol-rich foods, such as tea (catechins), coffee (chlorogenic acid), wine (resveratrol) and fruits, the information regarding the antioxidant potential of BSG is scarce. To the best of our knowledge, there are no published reports relating the phenolic composition and antioxidant activity of BSG obtained from different malt types. Recently, McCarthy et al. (2012) have investigated the ability of phenolic rich BSG extracts to protect against DNA damage in human lymphocytic U937 cells measured by the Comet assay. Their results showed that black BSG extracts, which had the highest phenol content, provided the greatest protection against $\mathrm{H}_{2} \mathrm{O}_{2}$-induced DNA damage.

The present study aims at evaluating the phenolic composition and antioxidant activity of BSG extracts obtained by microwaveassisted extraction (MAE). Light (pilsen, melano, melano 80 and carared) and dark (chocolate and black) malts were assessed for the total phenolic content (TPC) by the Folin-Ciocalteu assay, and for the antioxidant activity by the 2,2-diphenyl-1-picrylhydrazyl (DPPH), 2,2'-azino-bis(3-ethylbenzothiazoline-6-sulfonic acid) (ABTS) and 2-deoxyribose degradation assays. The phenolic compounds present in the BSG extracts were identified by HPLC-DAD/ESI-MS/MS.

\section{Materials and methods}

\subsection{Chemicals and reagents}

Folin-Ciocalteu's reagent (Merck, Darmstadt, Germany) and sodium carbonate (Sigma-Aldrich) were employed for the measurement of the total phenolic content (TPC). The calibration curve was constructed with gallic acid (GA, Sigma-Aldrich).

For the antiradical activity assessment, DPPH (Sigma-Aldrich) was used. The ABTS methodology employed ABTS and potassium persulfate $\left(\mathrm{K}_{2} \mathrm{~S}_{2} \mathrm{O}_{8}\right)$ purchased from Sigma-Aldrich. 2-deoxyribose assay was performed using $\mathrm{L}(+)$ ascorbic acid (Sigma-Aldrich), trichloroacetic acid (Riedel-de-Haën), 2-thiobarbituric acid, 2-deoxy-D-ribose, hydrogen peroxide $35 \%$ and $\mathrm{FeCl}_{3}$ (Fluka)

Ferulic acid (FA, 99\%) and $p$-coumaric acid ( $p$-CA, 98\%) standards were purchased from Sigma-Aldrich. Stock standard solutions $(500 \mathrm{mg} / \mathrm{L}$ ) of these compounds were prepared by rigorous dissolution of the analyte in methanol. Standard solutions were stored at $-20{ }^{\circ} \mathrm{C}$ and used for further dilutions. High-purity water from a Millipore Simplicity 185 water purification system (Millipore Iberian S.A.) was used for all chemical analyses and glassware washing. The solvents employed for HPLC analyses were filtered through a nylon filter of $0.45 \mu \mathrm{m}$ pore size (Whatman) and degassed for $10 \mathrm{~min}$ in an ultrasound bath.

\subsection{Samples}

BSG samples used throughout this work were kindly supplied by Unicer - Bebidas de Portugal, S.A. (S. Mamede de Infesta, Portugal) and Os Três Cervejeiros, Lda (Porto, Portugal), and were obtained from six malt types. Different malt types result from special malting procedures applied to a range of raw materials. These are usually grouped into three types based on the material used, namely, raw cereals, pale malt, or green malt (germinating cereal) (Guido \& Moreira, 2013). For a matter of facilitation, the different BSG extracts from the six malt types were divided into two main families, named here light malts (pilsen, melano, melano 80 and carared) and dark malts (chocolate and black). Pilsen malt is an exceptionally light colored 2-row base malt that produces a very light colored, clean, crisp wort and is used as a base malt for all beer styles. Caramel malt is made from green malt that is produced by drying the wet germinated barley at controlled temperatures, causing the starches to convert to sugars and caramelize. Melano malts are specialty malts slowly dried as the temperature is raised, allowing melanoidins to form as part of the kilning process. Chocolate malt shares many of the characteristics of black malt but, because it is roasted for a slightly shorter period and end temperatures are not so high, some of the harsher flavors of black malt are not so pronounced and EBC (European Brewing Convention) color is 200 units lighter. The specifications of each malt type, in particular color range and kilning temperature (KT) are listed in Table 1.

The BSG samples used for phenolic extraction correspond to the remaining solid fraction obtained following the removal of wort during the pilot scale production of beer in the brewing process. For wort production $25 \mathrm{~g}$ of pilsen malt was mixed with the same quantity of the colored malt and milled in a Bühler Miag disk mill. $200 \mathrm{~mL}$ of distilled water, at $45^{\circ} \mathrm{C}$, was added. After $30 \mathrm{~min}$, temperature was increased at $1{ }^{\circ} \mathrm{C} / \mathrm{min}$ for $25 \mathrm{~min}$ until it reached $70{ }^{\circ} \mathrm{C}$. The temperature was maintained at $70{ }^{\circ} \mathrm{C}$ for $1 \mathrm{~h}$. The mash was cooled, made up to $450 \mathrm{~g}$ with water and filtered. The obtained solid residue was frozen, lyophilized and then finely ground in a laboratory mill (Casella, London, UK) and sieved through a 35 -mesh $(\leq 0.5 \mathrm{~mm})$ sieve. The dried BSG samples were stored at $-20{ }^{\circ} \mathrm{C}$ until further use. Pilsen wort production was obtained using $50 \mathrm{~g}$ of pilsen malt, and following the same procedure.

\subsection{Microwave-assisted extraction}

BSG's phenolics were extracted according to the method previously optimized and reported by Moreira, Morais, Barros, Delerue-Matos, and Guido (2012). MAE was performed with a MARS-X 1500 W (Microwave Accelerated Reaction System for Extraction and Digestion, CEM, Mathews, NC, USA) configured with a 14 position carousel. Dried BSG sample $(1 \mathrm{~g})$ was transferred to the PTFE extraction vessels with $20 \mathrm{~mL}$ of $0.75 \% \mathrm{NaOH}$ concentration; then the vessels were closed. Extraction was performed during $15 \mathrm{~min}$ at $100{ }^{\circ} \mathrm{C}$ at maximum stirring speed. During operation, both temperature and pressure were monitored in a single vessel (control vessel). Magnetic stirring in each extraction vessel and a sensor registering the solvent leaks in the interior of the microwave oven were also used.

After extraction, vessels were allowed to cool at room temperature before opening and the extracts were then centrifuged for $15 \mathrm{~min}$ at $4000 \mathrm{rpm}$. The $\mathrm{pH}$ of the supernatant was adjusted to $\mathrm{pH} 6.5$ with $\mathrm{HCl}$ $6 \mathrm{M}$, and after filtration through a cellulose filter $(0.45 \mu \mathrm{m})$, the extracts were preserved at $-20{ }^{\circ} \mathrm{C}$ until further analysis.

\subsection{Determination of total phenolic content}

The TPC of the BSG extracts was determined by the Folin-Ciocalteu method as described by Dvorakova et al. (2008). In a test tube, $1 \mathrm{~mL}$ of diluted sample or standard solution and $5 \mathrm{~mL}$ of 10 -fold diluted Folin-Ciocalteu's phenol reagent were mixed. After 5 min of incubation, $4 \mathrm{~mL}$ of sodium carbonate solution $(7.5 \%, \mathrm{w} / \mathrm{v})$ was added and mixed well. After $2 \mathrm{~h}$ of incubation at room temperature in the dark, the absorbance was measured at $740 \mathrm{~nm}$ in a Shimadzu UV-3101 spectrophotometer (Kyoto, Japan). The total polyphenol concentration was calculated from a calibration curve, using GA as standard (5-150 mg/L). The results were expressed as mg GA equivalents

Table 1

Color and kilning temperatures for the six malt types used in the wort production.

\begin{tabular}{llcl}
\hline & Malt type & Color range/EBC units & Kilning temperatures $/{ }^{\circ} \mathrm{C}$ \\
\hline \multirow{2}{*}{ Light malts } & Pilsen & $3.5-5.7$ & $80-85$ \\
& Melano & $37-43$ & 130 \\
& Melano 80 & $75-85$ & 130 \\
& Carared & $90-360$ & $120-160$ \\
\multirow{5}{*}{ Dark malts } & Chocolate & $800-1000$ & 220 \\
& Black & $1350-1500$ & 230 \\
\hline
\end{tabular}


(GAE) per dry weight (DW) of BSG. All measurements were done in triplicate.

\subsection{Determination of antioxidant activity}

\subsubsection{DPPH radical scavenging activity assay}

DPPH is a stable free radical and has been commonly used to screen phenolic compounds containing high free radical scavenging ability. The DPPH radical scavenging activity of each extract was measured according to the method of Brand-Williams, Cuvelier, and Berset (1995) modified by Goupy, Hugues, Boivin, and Amiot (1999). DPPH radicals have an absorption maximum at $515 \mathrm{~nm}$, which disappears with reduction by an antioxidant compound. The DPPH solution in methanol $\left(6.6 \times 10^{-5} \mathrm{M}\right)$ was prepared daily and $2.85 \mathrm{~mL}$ of this solution was mixed with $150 \mu \mathrm{L}$ of the BSG extracts and vortexed. The absorbance decrease at $515 \mathrm{~nm}$ was measured after $120 \mathrm{~min}$ at room temperature in the dark. Lower absorbance of the reaction mixture indicates higher free radical scavenging activity. Results were expressed as antiradical power (ARP, \%) which is defined as $\left(1 / \mathrm{EC}_{50} \times 100\right)$, where $\mathrm{EC}_{50}$ is the quantity of extract capable of reducing the initial concentration of DPPH to one half. Data were reported as mean \pm standard deviation (SD) for three replicates.

\subsubsection{ABTS radical scavenging activity assay}

The free radical scavenging activity of BSG extracts was also evaluated using the ABTS radical cation decolorization assay (Dudonné, Vitrac, Coutiére, Woillez, \& Mérillon, 2009), which is based on the reduction of ABTS radicals by antioxidants of the BSG extracts. The stock solutions included $7 \mathrm{mM}$ ABTS solution and $2.45 \mathrm{mM} \mathrm{K}_{2} \mathrm{~S}_{2} \mathrm{O}_{8}$ solution. The working solution was then prepared by mixing the two stock solutions in equal quantities and allowing them to react in the dark for $16 \mathrm{~h}$ at room temperature to produce a dark green solution. Prior to the assay, the ABTS solution was diluted in deionized water to obtain an absorbance of $0.70 \pm 0.02$ units at $734 \mathrm{~nm}$. Then BSG extracts or standards $(150 \mu \mathrm{L})$ were allowed to react with $2850 \mu \mathrm{L}$ of the ABTS solution, and the absorbance reading was taken after $2 \mathrm{~h}$ at room temperature in the dark. A calibration curve was obtained by using trolox standard solutions at various concentrations $(0-100 \mathrm{mg} / \mathrm{L})$. The degree of ABTS radical-scavenging activity of BSG extracts was calculated, based on the trolox standard curve, and was expressed in terms of mg trolox equivalents (TE)/g DW of BSG. All solutions were prepared on the day that they were used and all measurements were carried out in triplicate.

\subsubsection{2-Deoxyribose assay}

The scavenging activity of BSG extracts towards hydroxyl radical was measured by the deoxyribose method with some modifications (Zhao et al., 2006). $100 \mu \mathrm{L}$ of BSG extract, $100 \mu \mathrm{L}$ of 1 mM EDTA, $100 \mu \mathrm{L}$ of $1 \mathrm{mM} \mathrm{FeCl}{ }_{3} 6 \mathrm{H}_{2} \mathrm{O}, 100 \mu \mathrm{L}$ of $36 \mathrm{mM}$ 2-deoxy-D-ribose, $100 \mu \mathrm{L}$ of $10 \mathrm{mM} \mathrm{H} \mathrm{O}_{2}$ and $100 \mu \mathrm{L}$ of $1 \mathrm{mM} \mathrm{L}(+)$-ascorbic acid were mixed and the final volume was adjusted to $1.0 \mathrm{~mL}$ with $25 \mathrm{mM}$ phosphate buffer (pH 7.4). After incubation at $37^{\circ} \mathrm{C}$ for $1 \mathrm{~h}$, the reaction was stopped by adding $1.0 \mathrm{~mL}$ of $10 \%$ TCA $(\mathrm{w} / \mathrm{v})$ and $1.0 \mathrm{~mL}$ of $1.0 \%$ TBA $(\mathrm{w} / \mathrm{v})$ in $0.05 \mathrm{M} \mathrm{NaOH}$. The mixture was heated in a water bath at $85^{\circ} \mathrm{C}$ for $30 \mathrm{~min}$. Once samples were cooled, the final volume was adjusted to $5.0 \mathrm{~mL}$ with deionized water. Absorbances were measured at $532 \mathrm{~nm}$ against water, and compared to control containing $100 \mu \mathrm{L}$ of deionized water instead of sample. Results were expressed as ARP (\%) which is defined as $\left(1 / \mathrm{EC}_{50} \times 100\right)$, where $\mathrm{EC}_{50}$ is the quantity of extract capable of reducing the initial concentration of hydroxyl radicals to one half. Data were reported as mean \pm SD for three replicates.

\subsection{Phenolic composition quantitative analysis}

The phenolic composition of the BSG extracts was analyzed by the HPLC method described by Rubilar, Pinelo, Shene, Sineiro, and Nuñez
(2007) with minor modification. The HPLC system (Jasco Corporation, Tokyo, Japan) consisted of a low pressure quaternary gradient unit (model LG-1580-04) with an in-line DG-1580-54 degasser and a model AS-950 auto-sampler. The system is equipped with a photodiode array detector (model MD-1510 UV/Vis multiwavelength detector).

Separation of polyphenols was achieved on a Phenomenex Synergi Hydro-RP $\mathrm{C}_{18}$ column ( $\left.150 \mathrm{~mm} \times 4.6 \mathrm{~mm}, 4 \mu \mathrm{m}\right)$ and a guard column with the same characteristics kept at room temperature. The chromatographic conditions were the following: flow rate $0.3 \mathrm{~mL} / \mathrm{min}$, sample injection volume of $20 \mu \mathrm{L}$ and mobile phase $\mathrm{A}$ (100\% methanol) and mobile phase B ( $0.1 \%$ formic acid). A gradient program was used as follows: $90 \%$ B in $0 \mathrm{~min}$, from $90 \%$ to $0 \%$ B in $110 \mathrm{~min}$, followed by $100 \% \mathrm{~A}$ for $20 \mathrm{~min}$ and back to $90 \% \mathrm{~B}$ in $10 \mathrm{~min}$ and $10 \mathrm{~min}$ of reconditioning before the next injection. The photodiode array detection was conducted by scanning between 190 and $600 \mathrm{~nm}$. Analytes in each sample were identified by comparing their retention times and UV-vis spectra with those of standard compounds. Peak purity was checked to exclude any contribution from interfering peaks.

The concentrations of individual phenolic compounds, in particular FA and $p$-CA, in BSG extracts were determined using external standard calibration curves in the concentration range of 5 to $200 \mathrm{mg} / \mathrm{L}$ (detection at $320 \mathrm{~nm}, n=7, \mathrm{r}^{2}=0.9997$ for FA; detection at $316 \mathrm{~nm}$, $n=7, \mathrm{r}^{2}=0.998$ for $p$-CA). Results were expressed in milligrams per $100 \mathrm{~g}$ of dry BSG (mg/100 $\mathrm{g}$ of DW).

\subsection{Identification of the major phenolic compounds}

The qualitative study of the phenolic composition in all samples was performed by HPLC coupled on-line with electrospray ionization (ESI) mass spectrometry. The HPLC system (Finnigan, Thermo Electron Corporation, San Jose, USA) consisted of a low-pressure quaternary pump (Finnigan Surveyor Plus) and an auto-sampler (Finnigan Surveyor Plus with 200-vial capacity sample). Separations were achieved in the same conditions as for HPLC-DAD analysis but the injection volume was $25 \mu \mathrm{L}$. A quadrupole ion trap mass spectrometer (Finnigan LCQ Deca XP Plus) equipped with an ESI source in the positive ion mode and Xcalibur software Version 1.4 (Finnigan) were used for data acquisition and processing. The interface conditions were applied as follows: capillary temperature, $325^{\circ} \mathrm{C}$; source voltage, $5.0 \mathrm{kV}$; capillary voltage, $4.0 \mathrm{~V}$; sheath gas $\left(\mathrm{N}_{2}\right)$ flow at 90 arbitrary units and auxiliary gas $\left(\mathrm{N}_{2}\right)$ flow rate at 25 arbitrary units. Data acquisition was performed between $\mathrm{m} / \mathrm{z} 100$ and 1000 . For the $\mathrm{MS}^{2}$ analyses an activation energy of $45 \%$ was applied. The positive ion mode was used in this study due to a better signal-to-noise ratio in comparison with the negative ion mode.

\subsection{Statistical analysis}

All data points are the mean and standard error values of at least three independent experiments. Differences in means were detected using one-way ANOVA and Tukey's test. The software employed for statistical analysis was Graphpad Prism, version 5 for Windows. The $p$-value less than $0.05(p<0.05)$ was considered statistically significant.

\section{Results and discussion}

\subsection{Total phenolic content}

After application of MAE method, the obtained BSG phenolic extracts were analyzed in terms of TPC by using the Folin-Ciocalteu method.

TPC from BSG extracts decreased with the increasing KT applied in the preparation of the different malt types (Table 1, Fig. 1A). BSG from the light malt types (pilsen, melano, melano 80 and carared; $\mathrm{KT} \leq 160{ }^{\circ} \mathrm{C}$, Table 1 ) were found to contain higher amounts of 
phenolic compounds when compared to the darker BSG's (chocolate and black; KT $\geq 200{ }^{\circ} \mathrm{C}$ ), $19.5 \pm 0.6$ and $16.2 \pm 0.6 \mathrm{mg} \mathrm{GAE} / \mathrm{g}$ DW BSG for light and dark types, respectively (Fig. 1A). In contrast to our results, McCarthy and co-workers (2012) recently reported that black BSG extracts exhibited the highest phenolic content. This difference can partially be explained by the type of malt used in the brewing process which has been shown to interfere with the content of individual phenolic compounds extracted (Dvořáková et al., 2008; Magalhães et al., 2011). In addition, this difference can also be due to the melanoidins generated during kilning by the Maillard reaction, whose content increases with KT. These compounds are widely reported to possess antioxidant properties (Inns, Buggey, Booer, Nursten, \& Ames, 2011), however other studies reported that melanoidins can trap polyphenols within its structure lowering the content of free phenolic compounds (Maillard \& Berset, 1995).

Among the BSG analyzed, pilsen type presents the highest phenolic content, $20 \pm 1 \mathrm{mg} \mathrm{GAE} / \mathrm{g} \mathrm{DW}$ (Fig. 1a). Kähkönen et al. (1999) studied the antioxidant capacity of 92 phenolic extracts from edible and nonedible plant materials (berries, fruits, vegetables, herbs, cereals, tree materials, plant sprouts, and seeds) and determined low phenolic content levels in cereals $(0.2-1.3 \mathrm{mg} / \mathrm{g}$ GAE). Those results were considerably lower than those obtained in the present work for BSG extracts. Recently, Meneses et al. (2013) also evaluated the efficiency of different solvent compositions (methanol, ethanol, acetone, hexane, ethyl acetate, water, methanol:water mixtures, ethanol:water mixtures, and acetone:water mixtures) for extracting antioxidant phenolic compounds from BSG and reported that the extract produced with $60 \% \mathrm{v} / \mathrm{v}$ acetone had the highest content of total phenols $(9.90 \mathrm{mg}$ $\mathrm{GAE} / \mathrm{g}$ ). Again a higher phenolic content was found in the pilsen BSG used in this work when compared with the results of Meneses et al. (2013). The higher values of phenolic content determined in the present work when compared with those from others works can be extensively discussed. The samples type and origin and the extraction technique used can be pointed as the main reasons for the verified differences. Although, it is possible to conclude that the pilsen BSG sample studied in this work and the extraction procedure are responsible for the highest values of phenolic content determined in this type of samples. In fact, the extraction technique is probably the most influential factor since BSG is a lignocellulosic material which contains significant amount of phenolic acids esterified to the cell-wall, making their recovery harder (Meneses et al., 2013; Moreira et al., 2012). Generally, the conventional extraction techniques, such as mechanical agitation solvent extraction, Soxhlet extraction, enzymatic and alkaline hydrolysis extractions (Dvorakova et al., 2008; Mussatto et al., 2007; Szwajgier, Waśko, Targoński, Niedźwiadek, \& Bancarzewska, 2010), applied for the recovery of these compounds are time and solvent consuming, submitting polyphenols to thermal degradation and decreasing the phenolic content of the extracts. Furthermore, BSG phenolic extraction by MAE procedure may be more efficient in breaking ether and ester bonds of lignin to phenolic compounds, allowing a higher amount of these compounds, which explains the main difference between our results and those reported in the literature.

\subsection{Ferulic and p-coumaric acids content}

The major compounds contributing to the phenolic content of BSG were FA and $p$-CA. For the six BSG analyzed it was found that FA occurs in higher amounts than p-CA (Fig. 2), which is in accord with
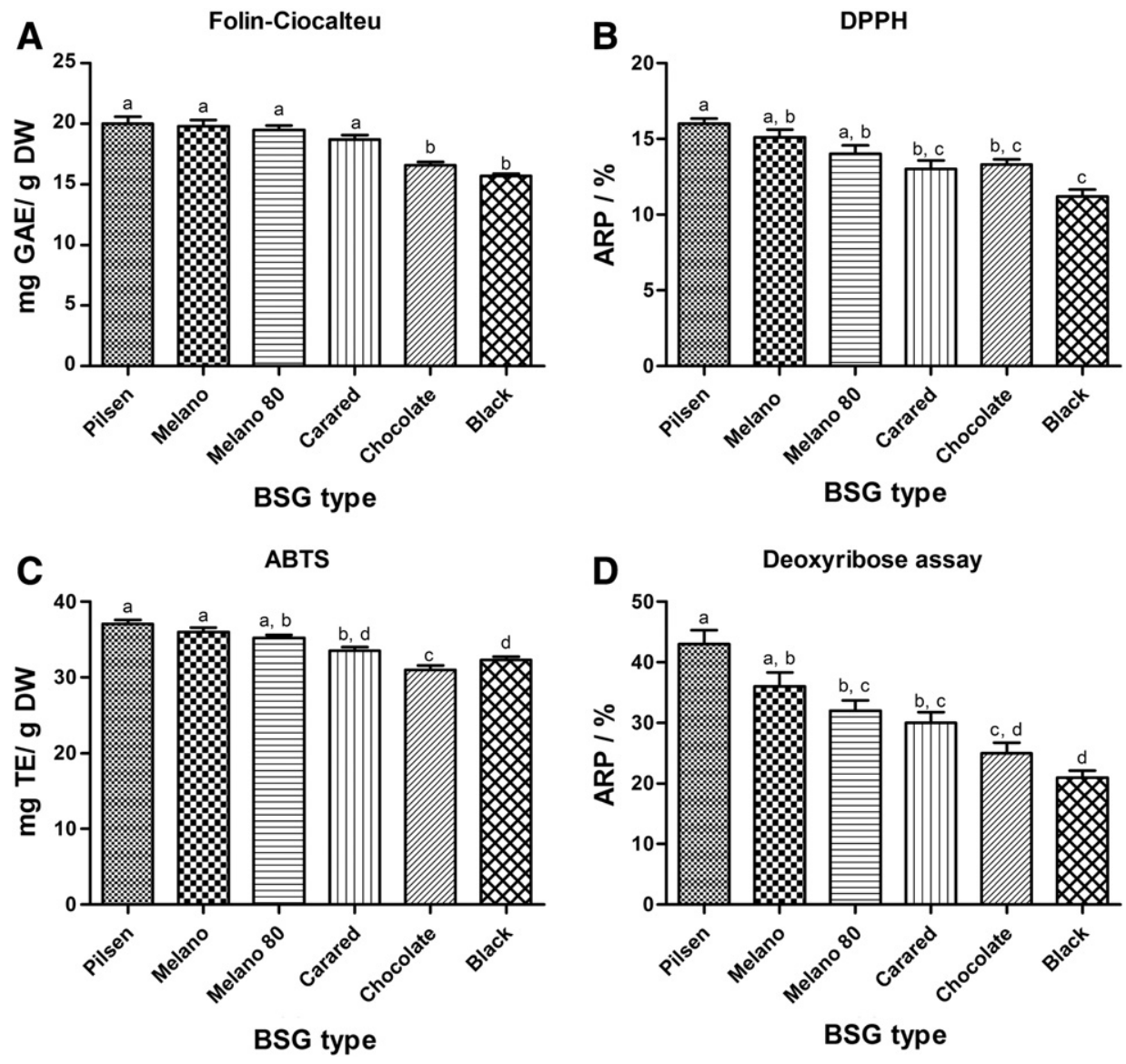

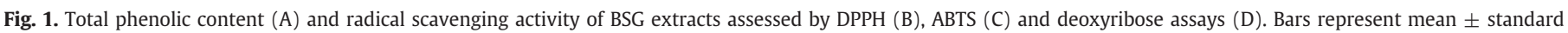
deviation. Bar means with different superscript letter are statistically significant $(p<0.05)$. GAE - gallic acid equivalents; TE - trolox equivalents. 
previous works (Bartolomé et al., 2002; Moreira et al., 2012; Mussatto et al., 2007). In the current study, the average of total FA and $p$-CA amounts of the light extracts was approximately 2-fold higher as compared to the dark extracts. The highest levels obtained for the light extracts were $149 \pm 7 \mathrm{mg} \mathrm{FA} / 100 \mathrm{~g}$ DW and $47 \pm 7 \mathrm{mg}$-CA/100 g DW (pilsen type) while those for the dark extracts were $77 \pm 3 \mathrm{mg}$ FA/100 g DW and $21 \pm 1 \mathrm{mg} p$-CA/100 g DW (black type). McCarthy and co-workers (2012) have also reported that the content of FA is significantly reduced for black extracts. As mentioned before, this can be attributed to the formation of melanoidins during kilning, mainly present in dark malts, which can trap polyphenols within its structure lowering the content of these phenolic compounds (Maillard \& Berset, 1995). Also, the thermal degradation with increasing KT can promote an overall decrease in the level of these hydroxycinnamic acids in dark BSG samples (Inns et al., 2011). Moreover, the higher content of these two acids in light BSG can also be due to the release of bound phenolics from cell walls by the action of ferulic acid esterase (FAE) and associated enzymes, which are more active at lower KT. As the temperature increases, the FAE becomes less active and the FA release is slowed. Samaras, Camburn, Chandra, Gordon, and Ames (2005) also reported that neither catechin nor FA were identified in black or chocolate malt or roasted barley (highly roasted malts).

The correlation analysis between the TPC and FA and p-CA contents in the BSG extracts $\left(r^{2}=0.9493\right.$ and 0.8038 , respectively) suggests that these two cinnamic acids are the major contributors for the higher TPC of pilsen BSG.

\subsection{Antioxidant properties of BSG extracts}

It is well known that the radical system used for antioxidant evaluation may influence the experimental results, and two or more radical systems are required to investigate the radical-scavenging capacities of a selected sample. Thus, in the present work, DPPH, ABTS and deoxyribose assays were used for the evaluation of the antioxidant activity of the extracts obtained from BSG samples. In this study, the extracts obtained from the six BSG displayed radical scavenging activity as shown in Fig. 1B, C and D.

The light extracts showed higher scavenging activities towards the radicals DPPH and ABTS than the dark extracts, which is in accordance with the higher TPC previously obtained $\left(r^{2}=0.7540\right.$ and 0.8129 for DPPH and ABTS, respectively). The DPPH radical-scavenging activity

\section{FA and $p-C A$ content}

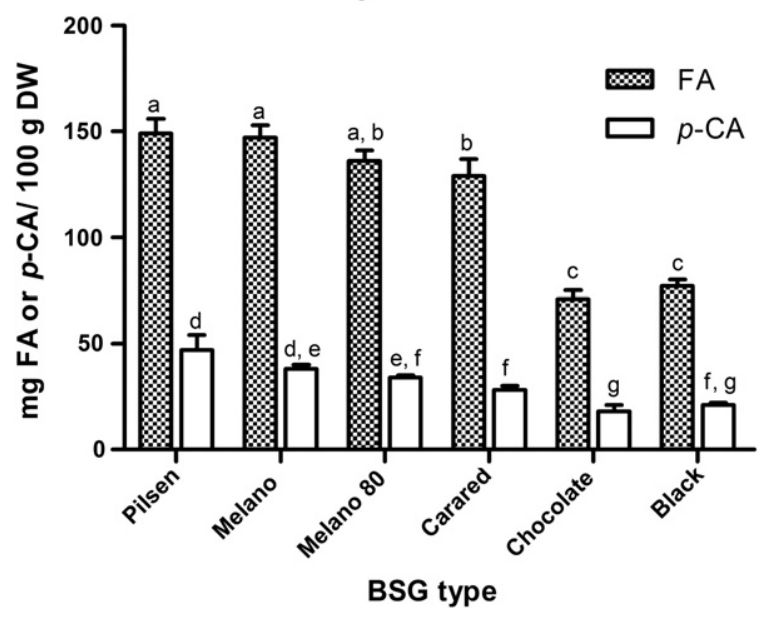

Fig. 2. Ferulic and p-coumaric acids content of BSG extracts. Bars represent mean \pm standard deviation. Bar means with different superscript letters are statistically significant $(p<0.05)$. decreases with increasing degree of malt color and KT (Table 1, Fig. 1B), with light extracts exhibiting higher DPPH radical-scavenging activity (15 \pm 1 and $12 \pm 1 \%$ for light and dark types, respectively). It has been reported that free radical-scavenging activity is greatly influenced by the phenolic composition of the sample. Therefore, this result could be anticipated because of the higher amounts of total phenolics and FA and $p$-CA contents present in the light BSG extracts.

The ABTS assay was also used for in vitro assessment of free radical activity and the relative ability to scavenge the ABTS radical has been compared with the standard trolox. Results were similar to those obtained for DPPH assay, with light BSG exhibiting a stronger ABTS cation radical-scavenging activity than dark BSG ( $35 \pm 2$ and $31.7 \pm$ $0.9 \mathrm{mg}$ TE/g DW for light and dark types, respectively) as shown in Fig. 1C.

The scavenging effect of hydroxyl radical was investigated using the 2-deoxyribose assay and the results are shown in Fig. 1D. The results were in agreement with those obtained for the DPPH and ABTS methods, with light BSG extracts having an antioxidant activity higher than dark BSG extracts ( $35 \pm 6$ and $23 \pm 3 \%$ for light and dark types, respectively). BSG extract from pilsen type exhibited the greatest scavenging effect of hydroxyl radicals among the BSG samples $(43 \pm 4)$. Vijayabaskar and Shiyamala (2012) determined the ARP by DPPH assay for the standard GA which is recognized for its antiradical properties. The value obtained (55\%) was closer to the ARP from pilsen BSG, confirming that this by-product exhibited good antiradical activity. Additionally, the results shown in Fig. 1D demonstrate that the difference in the ARP between light and dark BSG extracts assessed by the deoxyribose assay is higher than in the ARP measured by DPPH method. The average percentage for the difference was $20 \%$ for deoxyribose assay and only $5 \%$ for DPPH method. This variation may be caused by phenols, such as hydroxycinnamic acids, which are present in higher amount in light BSG extracts and are more prone to scavenge hydroxyl radicals than DPPH radicals. Significant positive correlations, $\mathrm{r}^{2}=$ 0.7851 and 0.9299 , were observed between FA and $p$-CA and antiradical efficiency determined using deoxyribose method, indicating that these phenolic compounds are the major contributors to the antiradical activity of these BSG extracts.

Briefly, the reported results suggest that BSG extracts from light malts (Table 1) were found to contain higher amounts of total and individual phenolics, such us FA and $p$-CA, which might be responsible for the strong antioxidant activity measured in several chemical assays. It was observed that the BSG containing higher levels of TPC also exhibited a strong antioxidant effect, which was statistically confirmed by correlation analysis $\left(r^{2}=0.7540,0.8129\right.$ and 0.8375 for $\mathrm{DPPH}, \mathrm{ABTS}$ and deoxyribose assays, respectively).

\subsection{Phenolic characterization}

The characterization of the MAE extract of BSG from industrial production by HPLC-DAD/ESI-MS/MS has previously revealed that this matrix was mainly composed of phenolic acids as well as several isomeric ferulate dehydrodimers and one dehydrotrimer (Moreira et al., 2012). In the present work, the same study was performed over the BSG extracts for pilsen, chocolate and black malts in order to examine the main differences in their phenolic composition, mainly in FA dimers. Comparing the MS results obtained for the three BSG samples analyzed in this study and BSG from industrial production major differences were not detected in the compounds identified. As expected, hydroxycinnamic acids ( $p$-CA (peak 4), FA (peak 5) and sinapic (peak 13)) were the main phenolic compounds in the BSG extracts. The structures of the other peaks identified in the chromatograms shown in Fig. 3 were the same as those already characterized by Moreira et al. (2012). Several FA dehydrodimers (DiFA) with a $[\mathrm{M}+\mathrm{H}]^{+}$ion at $m / z 387$ were identified, namely 8-8'-DiFA aryltetralin form (peak 2), 8-5'-DiFA benzofuran form (peak 3), 8-8'-DiFA (peak 7), 8-5'-DiFA (peak 8), 8-8'-DiFA cyclic form (peak 9), 5-5'-DiFA methylated form (peak 10), 8-5'-DiFA 

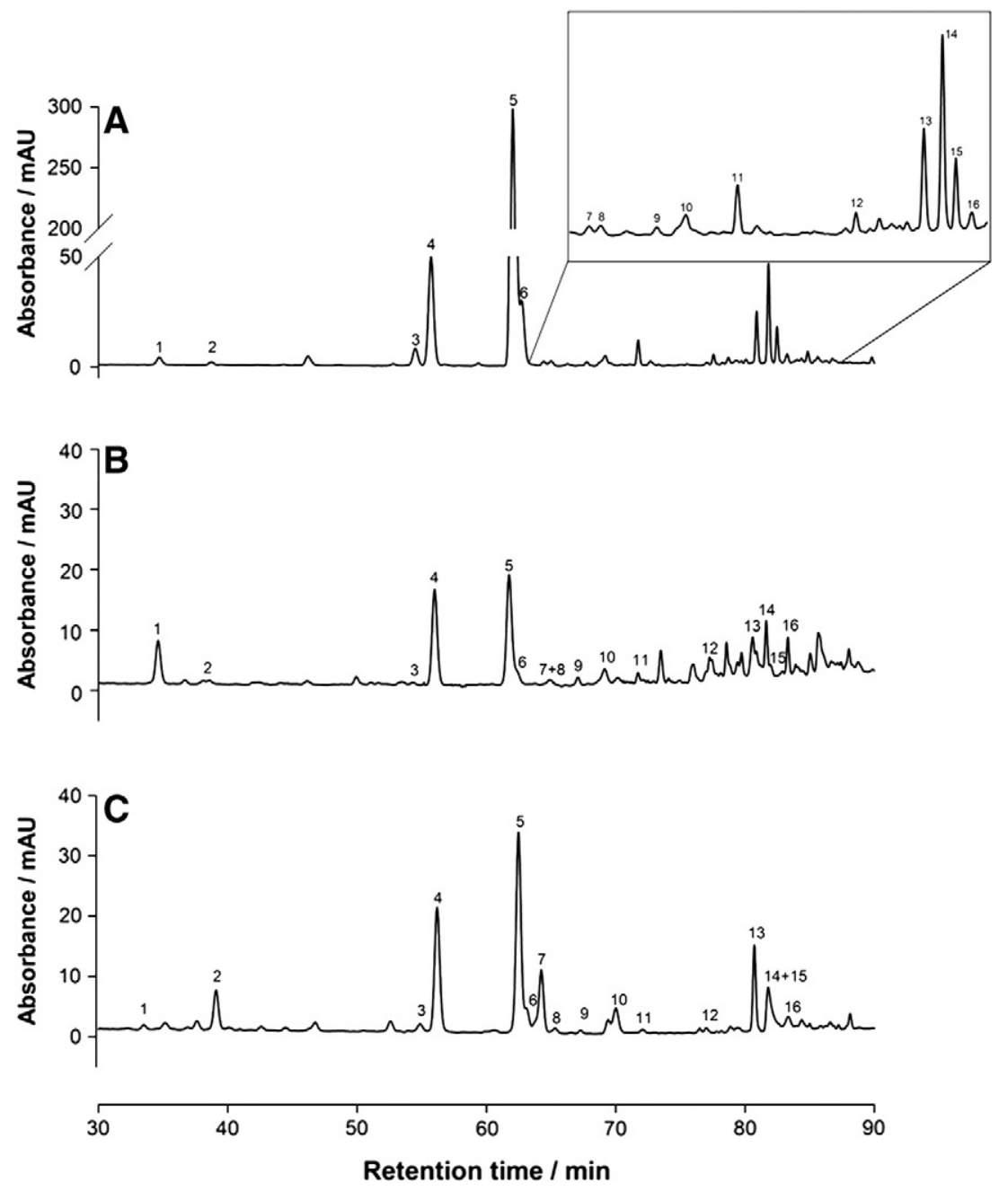

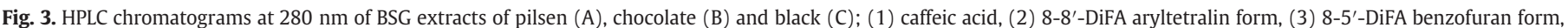

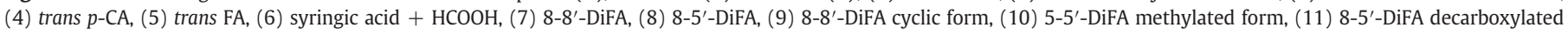
form, (12) unknown FA dehydrodimer, (13) sinapic acid, (14) 8-0-4'-DiFA, (15) 5-5', $8^{\prime}-0-4^{\prime \prime}-$ TriFA and (16) DiFA hydrated form.

decarboxylated form (peak 11) and 8-O-4'-DiFA (peak 14). These compounds were identified based on collision induced dissociation (CID) fragmentation (MS ${ }^{2}$ and $\mathrm{MS}^{3}$ spectra) in which subsequent loss of water $(\mathrm{m} / \mathrm{z} 369)$ and a carbonyl group $(\mathrm{m} / \mathrm{z} 341)$ were observed. This DiFA can be distinguished by their retention times and UV-spectra. Two other peaks (12 and 16) were also attributed to FA dehydrodimertype compounds, although final structure for peak 12 was not confirmed, while peak 16 corresponds to DiFA in hydrated form. The fragmentation pattern of the compound identified as peak 15 with a protonated molecule at $m / z 579$ corresponds to TriFA (5-5', $8^{\prime}-0-4^{\prime \prime}-$ TriFA). This parent ion resulted in two major fragments with $m / z 561$ and 533 , which is fully consistent with that previously observed for DiFA.

Although no differences were detected in the phenolic profile from pilsen, chocolate and black BSG, the content of some DiFA compounds was different. The peak areas obtained for the main DiFA identified in the three samples are reported in Table 2 . Among these compounds, 5-5'-DiFA in methylated form was the most abundant in the three studied BSG. Moreover, the pilsen BSG exhibited the highest total DiFA content, followed by the black and chocolate BSG. In fact, BSG types with higher FA content also showed higher levels of DiFA. ANOVA analysis showed significant differences $(p<0.05)$ among the three studied BSG types for the compounds that were studied except for 8-8'-DiFA in cyclic form (Table 2). This clearly indicates that the concentration of DiFA in BSG was influenced by the kilning regimes used in malting process.

\section{Conclusions}

Results from the present study showed that the type of malt used and the malting process, in particular the kilning regimes and roasting temperatures, may have important impact in terms of the phenolic composition and antioxidant features of BSG. Among the BSG studied, extracts from light types ( malt $\mathrm{KT} \leq 160{ }^{\circ} \mathrm{C}$ ) contained higher amounts of total and individual phenolic compounds, in particular FA and $p$-CA, than dark extracts (malt $\mathrm{KT} \geq 200{ }^{\circ} \mathrm{C}$ ). Although several classes of phenolic compounds were detected in all BSG samples, FA and $p$-CA were the most affected by the kilning regimes. This knowledge can

Table 2

Peak areas (expressed in $\mathrm{mAu}$ min) obtained at $280 \mathrm{~nm}$ of ferulic acid dehydrodimers in BSG extracts from pilsen, chocolate and black types.

\begin{tabular}{|c|c|c|c|c|}
\hline BSG type & $\begin{array}{l}8-5^{\prime}-\mathrm{DiFA}^{\mathrm{a}} \\
\text { benzofuran } \\
\text { form }\end{array}$ & $\begin{array}{l}\text { 8-8'-DiFA } \\
\text { cyclic } \\
\text { form }\end{array}$ & $\begin{array}{l}5-5^{\prime}-\mathrm{DiFA} \\
\text { methylated } \\
\text { form }\end{array}$ & $\begin{array}{l}\text { 8-5'-DiFA } \\
\text { decarboxylated } \\
\text { form }\end{array}$ \\
\hline Pilsen & $4.4 \pm 0.4^{\mathrm{a}}$ & $1.5 \pm 0.4^{c}$ & $4.0 \pm 0.3^{d}$ & $3.7 \pm 0.3^{f}$ \\
\hline Chocolate & $1.3 \pm 0.3^{b}$ & $1.5 \pm 0.4^{\mathrm{c}}$ & $2.2 \pm 0.3^{\mathrm{e}}$ & $1.6 \pm 0.2^{g}$ \\
\hline Black & $1.6 \pm 0.3^{b}$ & $1.2 \pm 0.4^{\mathrm{c}}$ & $4.0 \pm 0.2^{\mathrm{d}}$ & $1.2 \pm 0.3^{g}$ \\
\hline
\end{tabular}

Values are expressed as mean \pm standard deviation $(n=3)$. Means within a column with the same superscript letter are not significantly different $(p>0.05)$.

a DiFA: dehydrodiferulic acid. 
surely be valuable for exploring new applications for different types of BSG. It is evident from the results here reported that BSG extracts, especially from pilsen malt, can be used as an inexpensive and good natural source of functional substances for human health and the food industry.

\section{Acknowledgments}

This work has been supported by Fundação para a Ciência e a Tecnologia (FCT) through grant no. PEst-C/EQB/LA0006/2011, as well as by Santander (PP_IJUP2011 - 51). FCT is also acknowledged by MMM (SFRH/BD/60577/2009) and DOC (SFRH/BD/79939/2011) for their PhD studentships. The authors also thank Unicer - Bebidas de Portugal, S.A. and Os Três Cervejeiros, Lda for their support, including the supply of brewer's spent grain samples.

\section{References}

Aliyu, S., \& Bala, M. (2011). Brewer's spent grain: A review of its potentials and applications. African Journal of Biotechnology, 10(3), 324-331.

Barbosa-Pereira, L., Angulo, I., Paseiro-Losada, P., \& Cruz, J. M. (2013). Phenolic profile and antioxidant properties of a crude extract obtained from a brewery waste stream. Food Research International, 51(2), 663-669.

Bartolomé, B., \& Gómez-Cordovés, C. (1999). Barley spent grain: Release of hydroxycinnamic acids (ferulic and p-coumaric acids) by commercial enzyme preparations. Journal of the Science of Food and Agriculture, 79(3), 435-439.

Bartolomé, B., Santos, M., Jiménez, J. J., del Nozal, M. J., \& Gómez-Cordovés, C. (2002). Pentoses and hydroxycinnamic acids in brewer's spent grain. Journal of Cereal Science, 36(1), 51-58.

Brand-Williams, W., Cuvelier, M. E., \& Berset, C. (1995). Use of a free radical method to evaluate antioxidant activity. LWT- Food Science and Technology, 28(1), 25-30.

Dudonné, S., Vitrac, X., Coutiére, P., Woillez, M., \& Mérillon, J. -M. (2009). Comparative study of antioxidant properties and total phenolic content of 30 plant extracts of industrial interest using DPPH, ABTS, FRAP, SOD, and ORAC assays. Journal of Agricultural and Food Chemistry, 57, 1768-1774.

Dvořáková, M., Guido, L. F., Dostálek, P., Skulilová, Z., Moreira, M. M., \& Barros, A. A (2008). Antioxidant properties of free, soluble ester and insoluble-bound phenolic compounds in different barley varieties and corresponding malts. Journal of the Institute of Brewing, 114(1), 27-33.

Dvorakova, M., Moreira, M. M., Dostalek, P., Skulilova, Z., Guido, L. F., \& Barros, A. A. (2008). Characterization of monomeric and oligomeric flavan-3-ols from barley and malt by liquid chromatography-ultraviolet detection-electrospray ionization mass spectrometry. Journal of Chromatography. A, 1189(1-2), 398-405.

Goupy, P., Hugues, M., Boivin, P., \& Amiot, M. J. (1999). Antioxidant composition and activity of barley (Hordeum vulgare) and malt extracts and of isolated phenolic compounds. Journal of the Science of Food and Agriculture, 79(12), 1625-1634.

Guido, L. F., \& Moreira, M. M. (2013). Malting. In R. P. F. Guine, \& P. M. d. R. Correia (Eds.), Engineering Aspects of Cereal and Cereal-Based Products. : CRC Press.

Hashemi, M., Razavi, S. H., Shojaosadati, S. A., \& Mousavi, S. M. (2011). The potential of brewer's spent grain to improve the production of $\alpha$-amylase by Bacillus sp. KR-8104 in submerged fermentation system. New Biotechnology, 28(2), 165-172.

Inns, E. L., Buggey, L. A., Booer, C., Nursten, H. E., \& Ames, J. M. (2011). Effect of modification of the kilning regimen on levels of free ferulic acid and antioxidant activity in malt. Journal of Agricultural and Food Chemistry, 59(17), 9335-9343.

Kähkönen, M. P., Hopia, A. I., Vuorela, H. J., Rauha, J. P., Pihlaja, K., Kujala, T. S., et al. (1999). Antioxidant activity of plant extracts containing phenolic compounds. Journal of Agricultural and Food Chemistry, 47(10), 3954-3962.
Krishnaswamy, K., Orsat, V., Gariépy, Y., \& Thangavel, K. (2013). Optimization of microwave-assisted extraction of phenolic antioxidants from grape seeds (Vitis vinifera). Food and Bioprocess Technology, 6(2), 441-455.

Magalhães, P. J., Almeida, S. M., Carvalho, A. M., Gonçalves, L. M., Pacheco, J. G., Cruz, J. M., et al. (2011). Influence of malt on the xanthohumol and isoxanthohumol behavior in pale and dark beers: A micro-scale approach. Food Research International, 44(1), 351-359.

Maillard, M. N., \& Berset, C. (1995). Evolution of antioxidant activity during kilning: Role of insoluble bound phenolic acids of barley and malt. Journal of Agricultural and Food Chemistry, 43(7), 1789-1793.

Martins, S., Mussatto, S. I., Martínez-Avila, G., Montañez-Saenz, J., Aguilar, C. N., \& Teixeira, J. A. (2011). Bioactive phenolic compounds: Production and extraction by solid-state fermentation. A review. Biotechnology Advances, 29(3), 365-373.

McCarthy, A. L., O'Callaghan, Y. C., Connolly, A., Piggott, C. O., FitzGerald, R. J., \& O'Brien, N. M. (2012). Phenolic extracts of brewers' spent grain (BSG) as functional ingredients - Assessment of their DNA protective effect against oxidant-induced DNA single strand breaks in U937 cells. Food Chemistry, 134(2), 641-646.

Meneses, N. G. T., Martins, S., Teixeira, J. A., \& Mussatto, S. I. (2013). Influence of extraction solvents on the recovery of antioxidant phenolic compounds from brewer's spent grains. Separation and Purification Technology, 108, 152-158.

Moreira, M. M., Morais, S., Barros, A. A., Delerue-Matos, C., \& Guido, L. F. (2012). A novel application of microwave-assisted extraction of polyphenols from brewer's spent grain with HPLC-DAD-MS analysis. Analytical and Bioanalytical Chemistry, 403(4), 1019-1029.

Mussatto, S. I. (2009). Biotechnological potential of brewing industry by-products. In P. Nigam, \& A. Pandey (Eds.), Biotechnology for agro-industrial residues utilisation (pp. 313-326). The Netherlands: Springer.

Mussatto, S. I., Dragone, G., \& Roberto, I. C. (2007a). Ferulic and p-coumaric acids extraction by alkaline hydrolysis of brewer's spent grain. Industrial Crops and Products, 25(2), 231-237.

Mussatto, S. I., Fernandes, M., Dragone, G., Mancilha, I. M., \& Roberto, I. C. (2007b). Brewer's spent grain as raw material for lactic acid production by Lactobacillus delbrueckii. Biotechnology Letters, 29(12), 1973-1976.

Mussatto, S. I., Fernandes, M., Rocha, G. J. M., Órfão, J. J. M., Teixeira, J. A., \& Roberto, I. C. (2010). Production, characterization and application of activated carbon from brewer's spent grain lignin. Bioresource Technology, 101(7), 2450-2457.

Mussatto, S. I., \& Roberto, I. C. (2005). Acid hydrolysis and fermentation of brewer's spent grain to produce xylitol. Journal of the Science of Food and Agriculture 85(14), 2453-2460.

Naczk, M., \& Shahidi, F. (2004). Extraction and analysis of phenolics in food. Journal of Chromatography. A, 1054(1-2), 95-111.

Routray, W., \& Orsat, V. (2012). Microwave-assisted extraction of flavonoids: A review. Food and Bioprocess Technology, 5(2), 409-424.

Rubilar, M., Pinelo, M., Shene, C., Sineiro, J., \& Nuñez, M. J. (2007). Separation and HPLC-MS identification of phenolic antioxidants from agricultural residues: Almond hulls and grape pomace. Journal of Agricultural and Food Chemistry, 55(25), 10101-10109.

Samaras, T. S., Camburn, P. A., Chandra, S. X., Gordon, M. H., \& Ames, J. M. (2005). Antioxidant properties of kilned and roasted malts. Journal of Agricultural and Food Chemistry, 53(20), 8068-8074.

Szwajgier, D., Waśko, A., Targoński, Z., Niedźwiadek, M., \& Bancarzewska, M. (2010). The use of a novel ferulic acid esterase from Lactobacillus acidophilus K1 for the release of phenolic acids from brewer's spent grain. Journal of the Institute of Brewing, 116(3), 293-303.

Vijayabaskar, P., \& Shiyamala, V. (2012). Antioxidant properties of seaweed polyphenol from Turbinaria ornata (Turner) J. Agardh, 1848. Asian Pacific Journal of Tropical Biomedicine, 2(1), S90-S98.

White, J. S., Yohannan, B. K., \& Walker, G. M. (2008). Bioconversion of brewer's spent grains to bioethanol. FEMS Yeast Research, 8(7), 1175-1184.

Zhao, H., Dong, J., Lu, J., Chen, J., Li, Y., Shan, L., et al. (2006). Effects of extraction solvent mixtures on antioxidant activity evaluation and their extraction capacity and selectivity for free phenolic compounds in barley (Hordeum vulgare L.). Journal of Agricultural and Food Chemistry, 54(19), 7277-7286. 